\title{
Frequency choice of eRHIC SRF linac
}

\author{
W. Xu, I. Ben-Zvi, T. Roser, V. Ptitsyn
}

\section{Collider-Accelerator Department Brookhaven National Laboratory Upton, NY 11973}

\author{
U.S. Department of Energy \\ Office of Science, Office of Nuclear Physics
}

Notice: This document has been authorized by employees of Brookhaven Science Associates, LLC under Contract No. DE-SC0012704 with the U.S. Department of Energy. The United States Government retains a nonexclusive, paid-up, irrevocable, world-wide license to publish or reproduce the published form of this document, or allow others to do so, for United States Government purposes. 


\title{
FREQUENCY CHOICE OF ERHIC SRF LINAC
}

\author{
Wencan Xu, I. Ben-Zvi, T. Roser, V. Ptitsyn \\ Brookhaven National Lab, Upton, NY 11790
}

\begin{abstract}
eRHIC is a FFAG lattice based multipass ERL [1]. The eRHIC SRF linac has been decided to change from $422 \mathrm{MHz}$ 5-cell cavity to $647 \mathrm{MHz}$ 5-cell cavity. There are several considerations affecting the frequency choice for a high current multipassERL: the beam structure, bunch length, energy spread, beam-break-up (BBU) threshold, SRF loss considerations. Beyond the physics considerations, cost and complexity or risk is an important consideration for the frequency choice, especially when we are designing a machine to be built in a few years.

Although there are some benefits of using a $422 \mathrm{MHz}$ cavity for eRHIC ERL, however, there are some very critical drawbacks, including lack of facilities to fabricate a $422 \mathrm{MHz}$ 5-cell cavity, very few facilities to process such a cavity and no existing facility to test the cavity anywhere. As the cavity size is big and its weight is large, it is difficult to handle it during fabrication, processing and testing and no one has experience in this area. As the cavity size is large, the cryomodule becomes big as well. All of these considerations drive the risk of building eRHIC ERL with $422 \mathrm{MHz}$ cavities to a very high level. Therefore, a decision was made to change the frequency of main linac to be $647 \mathrm{MHz}$ 5-cell cavities.

This note will compare these two linacs: $422 \mathrm{MHz} 5$-cell cavity linac and $647 \mathrm{Mz} 5-$ cell cavity SRF linac, from both practical point of view and physics point of view.
\end{abstract}

\section{LOW FREQUENCY AND HIGH FREQUENCY CAVITY}

From physics point of view, there are a few benefits of using a low frequency SRF cavity for ERL [2], and there are also some drawbacks. For example,

(1) The transient voltage is inversely proportional to the square of frequency, thus favoring a lower frequency.

(2) The Lorentz detuning factor is inversely proportional to frequency so the LLRF control is easier at higher frequencies.

(3) The loss factor (wakefield) is inversely proportional to the aperture (for a constant ratio of the bunch length and frequency), thus favoring lower frequencies in term of induced energy spread and HOM power generation.

(4) The transverse beam-break-up (BBU) threshold is approximately inversely proportional to the cavity frequency and the linac length, favoring a lower frequency.

(5) With the same real estate and linac energy, more cavities are required for the higher frequency linac, so the part count is also higher, driving the cost up with frequency.

(6) One of the drawbacks for a lower frequency cavity is that N-doping will not improve the quality factor of a $422 \mathrm{MHz}$ cavity because at this low frequency, the cavity's quality factor is already dominated by the residual resistance. 
However, experiment results from Fermi Lab shows that the quality factor of $650 \mathrm{MHz}$ cavity improve a factor of 3 [3].

As addressed above, the $422 \mathrm{MHz}$ SRF linac brings various benefits for machine performance. However, from practical/project point of view, there is a large risk of using a $422 \mathrm{MHz}$ 5-cell cavity SRF linac. Until now, most elliptical SRF cavities, made by traditional technologies (deep-drawing and electron-beam wielding), were at a frequency higher than the $650 \mathrm{MHz}$ for the Project-X. The single-cell $500 \mathrm{MHz}$ cavities for light sources were made by spinning or hydroforming. In the USA, there is only one example of a single-cell $400 \mathrm{MHz}$ SRF cavity, which was made by JLab last year as the prototype of 3-cell SRF cavity for the BES-CLS project [4]. They encountered various difficulties at almost every step of the cavity fabrication and processing, mostly because of its big size and heavy weight. The vertical test result of this single-cell $400 \mathrm{MHz}$ prototype cavity showed that it quenched at $8 \mathrm{MV} / \mathrm{m}\left(\mathrm{Q} 0\right.$ was $3 \cdot 10^{9}$ at $4.2 \mathrm{~K}$ ) and the reason for quench is still not clear, although it is suspected to be due to electron-beam welding of the cavity's equator. For comparison, the design of the eRHIC ERL requires SRF cavities to operate at $18.5 \mathrm{MV} / \mathrm{m}$ with a $\mathrm{Q}_{0}$ of $3 \cdot 10^{10}$. In contrast, the existing facilities (over the world) are able to handle 5-cell $647 \mathrm{MHz}$ cavity, including cavity fabrication, processing and tests. At BNL, we made $3704 \mathrm{MHz}$ 5-cell cavities [5,6,7,8], which is very close to $647 \mathrm{MHz}$ 5-cell cavity in terms of cavity, our facilities (BCP, HPR, high temperature bake oven and vertical test facility) were commissioned. Another practical point is the RF amplifier [9], the efficiency of the RF amplifiers between $650 \mathrm{MHz}$ and $422 \mathrm{MHz}$ is not different, but a $50 \mathrm{~kW} 650 \mathrm{MHz}$ RF amplifier is more conventional than a $50 \mathrm{~kW} 422$ $\mathrm{MHz}$ RF amplifier.

From a SRF linac point of view, if the real-estate gradient is the same, the high frequency linac is cheaper because of the cryomodule size is smaller, but extra costs may incurred due to the increase part count of tuners and couplers. The cryomodule size is particularly important for eRHIC because of the limit of the existing RHIC tunnel.

Overall, the drawbacks of a $647 \mathrm{MHz}$ 5-cell cavity linac may be compensated by other means. However, the risk of the $422 \mathrm{MHz}$ choice is dramatically high because of the lack of fabrication experience and facilities to deal with it. Therefore, the development of 422 $\mathrm{MHz}$ SRF cavities requires a large investment in infrastructure. At the known frequency $647 \mathrm{MHz}$ cavity SRF, the risk is largely alleviated.

\section{FUNDAMENTAL MODE}

The cavity was initially designed with the frequency of $422 \mathrm{MHz}$, the 5-cell $647 \mathrm{MHz}$ cavity is scaled from the original design, so the fundamental mode's performance is the same. The $647 \mathrm{MHz}$ 5-cell cavity is named as BNL4 cavity. However, the cavity's length reduces from $2.56 \mathrm{~m}$ to $1.68 \mathrm{~m}$. The fundamental mode's performance of BNL4 cavities is listed in Table I. Figure 1 (top) shows Superfish model of the 647 BNL4 cavity. The field profile of the fundamental mode by Superfish is shown in Figure 1 (bottom). 

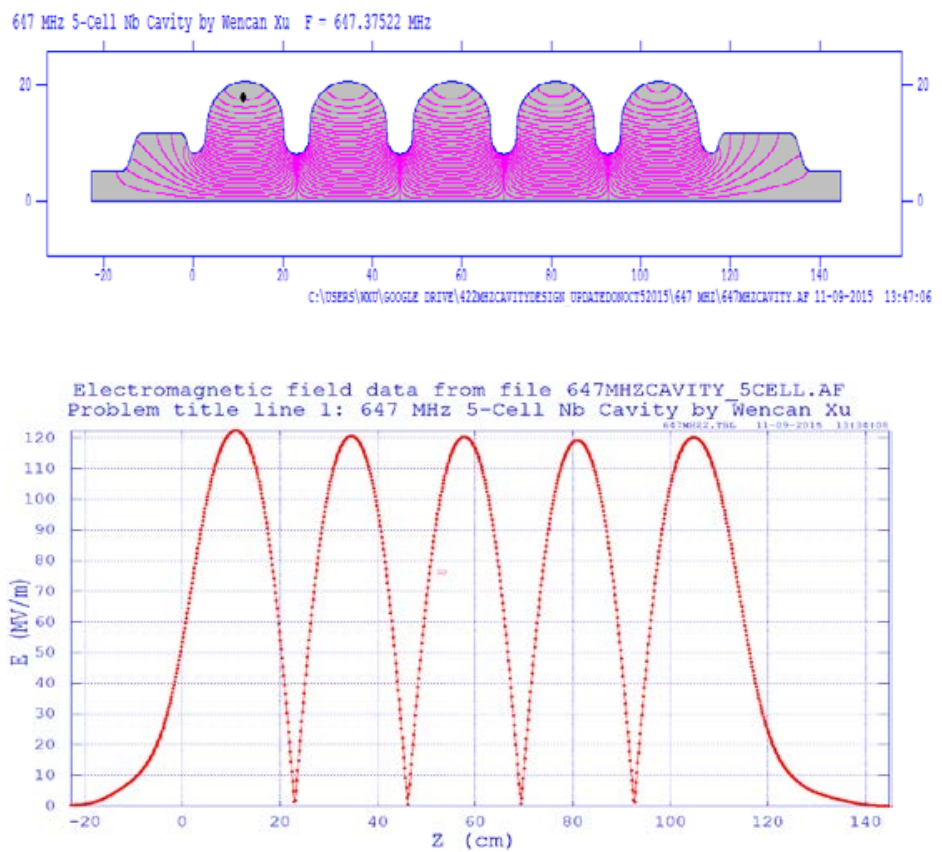

Figure 1: BNL4 cavity configuration (top) and fundamental mode field profile (bottom).

Table II: RF parameters of the BNL4.

\begin{tabular}{|c|c|c|}
\hline Parameters & $422 \mathrm{MHz}$ 5-cell cavity & $647 \mathrm{MHz}$ 5-cell cavity \\
\hline Frequency [MHz] & 647 & 647 \\
\hline Number of cells & 5 & 5 \\
\hline $\begin{array}{l}\text { Geometry factor } \\
{[\Omega]}\end{array}$ & 273 & 273 \\
\hline$(\mathrm{R} / \mathrm{Q}) /$ Cavity $[\Omega]$ & 502 & 502 \\
\hline Epeak/Eacc & 2.27 & 2.27 \\
\hline $\begin{array}{l}\text { Bpeak/Eacc } \\
{[\mathrm{mT} / \mathrm{MV} / \mathrm{m}]}\end{array}$ & 4.42 & 4.42 \\
\hline $\begin{array}{l}\text { Coupling factor } \\
{[\%]}\end{array}$ & 2.8 & 2.8 \\
\hline Cavity length [m] & 2.56 & 1.68 \\
\hline
\end{tabular}

\section{MONOPOLE MODES AND HOM POWER}

An average monopole mode HOM power generated by a single bunch travelling through a cavity is proportional to the bunch charge $Q_{b}$, beam current $I_{b}$, and the longitudinal loss factor $k_{p}$ :

$$
P_{\mathrm{ave}}=k_{\square} I_{\mathrm{b}} Q_{\mathrm{b}}
$$


As the average HOM power is linear with the loss factor. The loss factor should be an important optimization factor for high current ERL machine. Calculated by ABCI [10], the loss factor for a $3 \mathrm{~mm}$ (rms) bunch excluding the fundamental mode was $2.55 \mathrm{~V} / \mathrm{pC}$, which can be handled by the reduction of the number of eRHIC ERL passes. As the bunch length for the eRHIC design may change, various bunch lengths were used to calculate for $647 \mathrm{MHz}$ 5-cell cavity, which is shown in Figure 2.

With the nominal eRHIC beam parameters (7 passes at the maximum beam current of $26 \mathrm{~mA}$ ERL, $3 \mathrm{nC}$ per bunch) for an intermediate energy, where the HOM power is maximal, an average value of monopole mode HOM power in one BNL4 cavity is $2.78 \mathrm{~kW}$ per cavity. However, this power can be higher or lower if one considers the beam spectrum. This presents a big challenge for removing it out of the cryostat and it has to be damped outside the cryomodule, so bunch pattern should be optimized for HOM power during the ERL design.

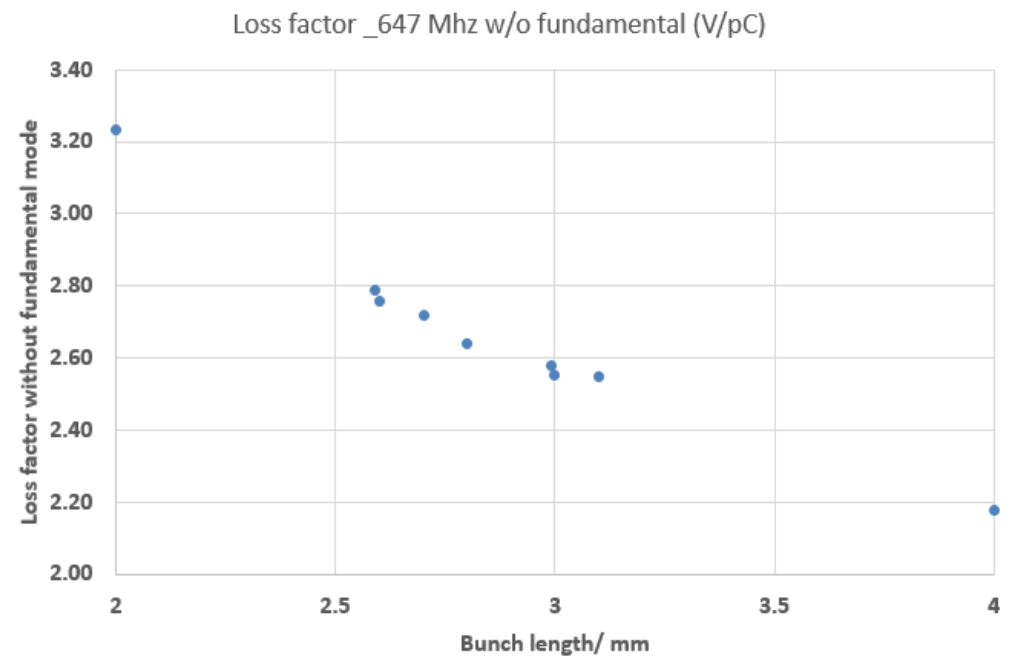

Figure 2: Integrated loss factors of the $647 \mathrm{MHz}$ 5-cell BNL4 cavity for different rms bunch length.

\section{TRANSVERSE BEAM-BREAK-UP (BBU)}

The FFAG lattice based eRHIC design is a multi-pass (up to 12), high current ERL design. One important concern for the linac cavity design is to increase the beam break up (BBU) threshold current. The transverse BBU threshold beam current depends mainly on the strength of dipole HOMs. Assuming that HOMs behave independently and do not interfere with each other, the threshold current in the presence of a single HOM can be approximated as [11]

$$
I_{t h}=-\frac{2 p c}{e k\left(R_{d} / Q\right) Q_{e x t} M_{12} \sin \left(\omega T_{r}\right)}
$$

Where $\mathrm{p}$ is the particle momentum, $\mathrm{c}$ is the speed of light, e is the electron charge, $\mathrm{k}$ is the higher-order-mode's wave number, $\mathrm{R}_{d} / \mathrm{Q}$ is the shunt impedance and $\mathrm{Q}_{\text {ext }}$ is the quality factor, M12 is the transport matrix parameter, and $T_{r}$ is the bunch return time. From the threshold current formula (2), it is clear that a small $R_{d} / Q$ and/or $Q_{\text {ext }}$ can 
increase the threshold current. A smaller $Q_{\text {ext }}$ means shorter damping time and larger current needed to deposit enough energy to disturb the beam.

One should notice that this formula fits for one cavity, one pass case. Although it is inversely proportional to the frequency, the threshold current reduces, in the worst case approximately as the square of number of passes. BBU code simulation shows that the threshold current of BNL4 cavity for eRHIC has at least a factor of 4 above the operation beam current, for a zero frequency spread due to fabrication (usually it is around a few $\mathrm{MHz}$ spread) in the HOM spectrum.

\section{MECHANICAL DESIGN AND PROTOTYPE CAVITY}

In eRHIC design, the electron beams will collide with different proton energies from $40 \mathrm{GeV}$ to $250 \mathrm{GeV}$, which corresponds to a frequency shift up to $174 \mathrm{kHz}$ for $647 \mathrm{MHz}$ cavity. ANSYS simulation shows that the cavity's tuning sensitivity is 84 $\mathrm{kHz} / \mathrm{mm}$, so the tuning range requirement for BNL4 cavity is $2 \mathrm{~mm}$. With a $4 \mathrm{~mm}$ thickness of $\mathrm{Nb}$ sheet, the cavity can be tuned up to $2.0 \mathrm{~mm}$ without exceeding the yield strength of Nb: 7000 psi, which is shown in Figure 5.

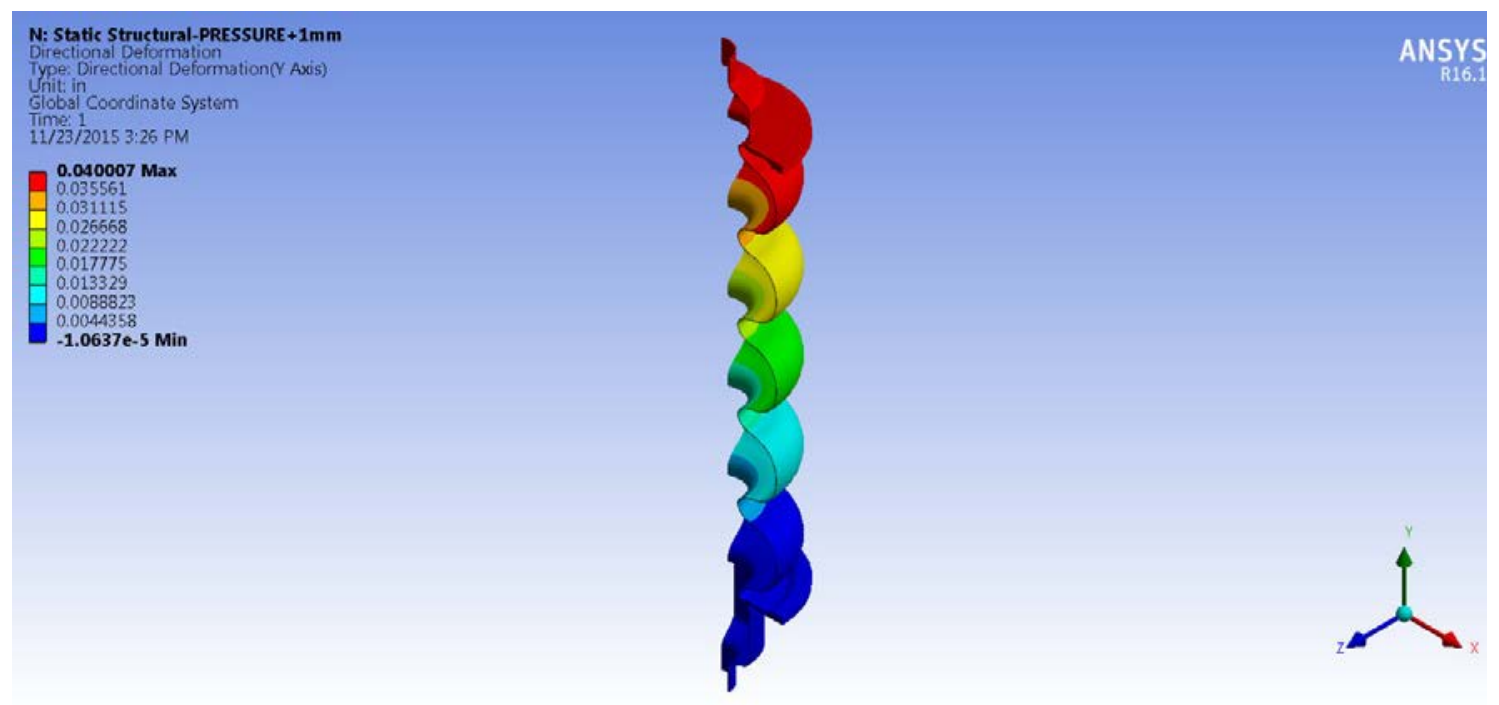

Figure 5: 5-cell prototype of the BNL4 cavity, 4mm thick without stiffness ring.

\section{SUMMARY}

The first part of this paper describes the frequency considerations for the eRHIC ERL. Although there are a few physics benefits by using a low frequency (422 MHz 5-cell cavity) linac, there are critical practical issues that increase the risk of the project. So, 647 MHz 5-cell cavity is the baseline of the eRHIC SRF ERL. The second part of this paper discusses the physics and mechanical design of the 5-cell $647 \mathrm{MHz}$ cavity. 


\section{REFERENCES}

1. eRHIC, "eRHIC Design Study: An Electron-Ion Collider at BNL", arXiv:1409.1633, December 2014.

2. S. Belomestnykh, et al., "On the frequency choice for the eRHIC SRF linac," IPAC2014, p. 1547

3. Anna Grassellino, "Status of $650 \mathrm{MHz}$ cavities for PIP-II" PIP-II Machine Advisory Committee, 9-11 March 2015

4. F. S He, et al, "A prototype cavity for ICS $\mathrm{x}$ - ray light source applications" http://accelconf.web.cern.ch/AccelConf/SRF2013/talks/thp014_talk.pdf”, SRF 2013

5. R. Calaga, Ph.D Thesis, http://www.agsrhirhichome.bnl.gov/people/rcalaga/html

6. Wencan Xu, I. Ben-Zvi, R. Calaga, H. Hahn, E.C. Johnson, J. Kewish, "High current cavity design at BNL”, Nucl. Instr. and Meth. A 622 (2010) 17-20.

7. Wencan $\mathrm{Xu}$, et al, "Progress on the high-current $704 \mathrm{MHz}$ superconducting RF cavity at BNL”, Proc. IPAC2012, pp. 2486-2488.

8. Wencan Xu, et al, "Vertical test results of $704 \mathrm{MHz}$ BNL3 SRF cavities", Proc. LIAC2012.

9. Private communication with Alexander Zaltsman.

10. ABCI, Azimuthal Beam Cavity Interaction, http://abci.kek.jp/

11. G. H. Hoffstaetter and I. V. Bazarov, Phys. Rev. ST Acecel Beams 7, 054401 (2004). 